Itinéraires Itinéraires

Littérature, textes, cultures

2011-1| 2011

Les Mémoires, une question de genre?

\title{
Les Mémoires de Madame Roland : être femme dans la tourmente de l'Histoire
}

Anne Coudreuse

\section{(2) OpenEdition}

1 Journals

Édition électronique

URL : https://journals.openedition.org/itineraires/1599

DOI : 10.4000/itineraires.1599

ISSN : 2427-920X

Éditeur

Pléiade

Édition imprimée

Date de publication : 1 avril 2011

Pagination : $29-43$

ISBN : 978-2-296-13692-2

ISSN : $2100-1340$

Référence électronique

Anne Coudreuse, «Les Mémoires de Madame Roland : être femme dans la tourmente de l'Histoire », Itinéraires [En ligne], 2011-1 | 2011, mis en ligne le 01 avril 2011, consulté le 28 juin 2022. URL : http:// journals.openedition.org/itineraires/1599; DOI : https://doi.org/10.4000/itineraires.1599

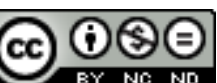

Itinéraires est mis à disposition selon les termes de la licence Creative Commons Attribution - Pas d'Utilisation Commerciale - Pas de Modification 4.0 International. 


\title{
Les Mémoires de Madame Roland : être femme dans la tourmente de l'Histoire
}

\begin{abstract}
Mme Roland's Mémoires are a hybrid of public and private, of History and intimacy. She wrote them during her incarceration in 1793. They are divided into two very distinct parts: the strictly historical Memoirs on the one hand and, on the other hand, the "Mémoires particuliers," which chronicle the private life of the Parisian bourgeoisie from 1760 to 1780 . This article explores the implications of the author's gender in "Mémoires historiques," since the memoirist is a woman, and what is more, she is the wife of a minister, who was criticized for allowing himself to be influenced by her and she was subjected to very violent attacks by the enemies of Roland.
\end{abstract}

Keywords : History, the French Revolution, girondins, femininity, politics

Mots clés : Histoire, Révolution française, girondins, féminité, politique

Les Mémoires de Mme Roland se situent à la croisée du public et du privé, de l'Histoire et de l'intime. Elle les a rédigés lors de son incarcération à la prison de l'Abbaye en 1793. Ils sont divisés en deux parties très distinctes : d'une part des Mémoires proprement historiques (Notices historiques, Interrogatoire de Mme Roland, Portraits et anecdotes, Brissot, Danton, Premier ministère, Second ministère, Seconde Arrestation, Anecdotes); d'autre part des "Mémoires particuliers » qui sont une chronique de la vie privée de la moyenne bourgeoisie parisienne entre 1760 et 1780 , et fournissent un document socio-historique précieux sur la sensibilité, les mœurs, les idées et le goût à la fin de l'Ancien Régime, tout en permettant d'entrer dans l'intimité de la jeune Marie-Jeanne dite Manon Phlipon. À cet ensemble s'ajoute un texte intitulé « Mes dernières pensées », rédigées à la prison de Sainte-Pélagie après le 2 octobre 1793 et l'anéantissement du parti girondin, alors que Mme Roland a pris la décision de se laisser mourir de faim. Dans l'édition du «Temps retrouvé » procurée au Mercure de France par Paul de Roux, les Mémoires historiques occupent 257 pages, les « Mémoires particuliers » 218 pages, et l'ensemble de 
textes regroupés sous le titre «Mes dernières pensées » 49 pages. Cette bipartition entre vie privée et Histoire (événements et portraits) peut s'articuler à la question du genre sexuel dans ces textes. En tant que femme de ministre, Mme Roland a joué un rôle politique important, car le couple avait, avant d'entrer en politique, l'habitude d'un travail commun. Mais Mme Roland, par l'éducation soignée qu'elle a reçue, est une femme à part, qui n'a pas attendu son mariage pour développer sa pensée et son goût de l'écriture et de la réflexion. Elle a même écrit en 1777 un discours pour l'Académie de Besançon, Comment l'éducation des femmes pourrait contribuer à rendre les hommes meilleurs, inspiré du Discours sur l'inégalité de Rousseau, où elle affirme le rôle civilisateur de la femme, dont l'autonomie est le moteur du progrès collectif, et permet d'éviter cette « rouille de barbarie » qui couvre les sociétés musulmanes. Nous limiterons notre corpus d'étude aux Mémoires historiques proprement dits, réservant pour un travail ultérieur une analyse de l'articulation entre vie privée et vie publique dans les « Mémoires particuliers », et de la façon très rousseauiste dont Mme Roland fait entrer le lecteur dans l'intimité de sa jeunesse et de sa formation, sans jamais oublier le fracas de l'Histoire. Nous voulons ici nous arrêter sur les implications pour la narratrice et pour son public, dans cet objet d'écriture qui tient du plaidoyer et du réquisitoire, du fait que la mémorialiste est une femme, et qui plus est, l'épouse d'un ministre à qui on a reproché de s'être laissé influencer par elle, Madame Roland ayant été l'objet d'attaques très violentes de la part des ennemis de Roland.

Dès les premières pages des Mémoires intervient un récit où se mêlent la dimension publique, historique, et la dimension privée et même intime, aussi bien dans le compte rendu de certains malaises du corps que dans l'évocation cryptée de l'amour pour Buzot qui pousse Mme Roland à s'éloigner de lui. Elle vient d'évoquer l'insurrection du 31 mai, et raconte, dans un dispositif textuel assez complexe, comment elle a cherché à fuir :

Je me préparais à faire viser à la municipalité des passeports au moyen desquels je devais me rendre avec ma fille à la campagne, où m'appelaient mes affaires domestiques, ma santé et beaucoup d'autres bonnes raisons; je calculais, entre autres, combien il serait plus facile à Roland seul de se soustraire à la poursuite de ses ennemis s'ils en venaient aux derniers excès, qu'il ne le serait à sa petite famille réunie; la sagesse voulait diminuer le nombre des points par lesquels il pouvait être accessible. (p. 49)

Mais elle ajoute aussitôt une note qui éclaire d'un jour différent cette décision tactique et ce sacrifice pour son mari :

Ce n'était pas ma plus forte raison; car, ennuyée du train des choses, je ne craignais rien pour moi; innocente et courageuse, l'injustice pouvait m'atteindre sans me flétrir; la subir était une épreuve que j'avais quelque 
plaisir à défier; mais une autre raison, que j'écrirai peut-être un jour et qui est toute personnelle, me décidait au départ.

C'est alors à l'éditeur, Paul de Roux, d'ajouter une note : "Mme Roland voulait quitter Paris pour retrouver, loin de Buzot, la paix du cœur. » On voit comment le politique, son vocabulaire et ses stratégies, sont pour ainsi dire contaminés ou rattrapés par le privé, comme le soulignent les expressions " mes affaires domestiques », qui renvoient à la maison et à sa bonne gestion, " ma santé », qui est l'expression de l'intimité du corps, et plus encore l'adjectif « personnelle », qui, associé à « raison », nous conduit à l'opposé de la raison d'État que certains préjugés voudraient voir prévaloir dans un récit historique. Cette façon oblique de dire sans dire son amour pour un autre, au moment même où elle affirme son dévouement à son mari en danger, est une manière de respecter un certain pacte de sincérité qui vaut aussi pour les Mémoires, mais plutôt dans un rapport de soi à soi, que de soi au lecteur, du moins à celui de la postérité, qui aura besoin des clés fournies par le premier cercle des lecteurs, amis des Roland, dépositaires des manuscrits de Mme Roland aussi bien que des secrets de son cœur. Avec un grand sens de la mise en scène où le malaise du corps intervient comme un fatum des temps modernes et empêche Mme Roland de fuir Paris, elle détaille les raisons publiques et privées qui entraînent son arrestation :

\footnotetext{
Mes passeports avaient été retardés à la section par les chicanes des zélés maratistes, aux yeux desquels j'étais suspecte; ils ne faisaient que de m'être délivrés, lorsqu'une attaque de coliques nerveuses, accompagnées d'horribles convulsions, seule indisposition que je connaisse et à laquelle m'exposent les vives affections d'une âme forte commandant à un corps robuste, m'obligea à garder le lit. (p. 49)
}

Alors que six hommes armés munis d'un ordre du comité révolutionnaire ont voulu arrêter Roland, elle décide de dénoncer ce fait à la Convention avec éclat : " en communiquer le projet à mon mari, faire une lettre au président et partir, fut l'affaire de quelques minutes » (p. 51). Elle reconnaît elle-même le rôle actif qu'elle a eu à ce moment, en particulier dans la rédaction de la lettre, et dans ce plaidoyer que constituent ces Mémoires, qui sont une tentative permanente d'autojustification, le lecteur aura du mal à la croire, quand elle cherche à cantonner son rôle à celui d'une maîtresse de maison silencieuse, prêtant seulement sa plume à Roland, comme elle l'a toujours fait depuis leur mariage en 1780. Ennemis du moment comme Danton, ou admirateurs du siècle suivant comme Sainte-Beuve, ne s'y sont d'ailleurs pas trompés. Élu député de Paris, Danton avait opté pour l'Assemblée et quitté le Conseil. Les électeurs de la Somme s'étant avisés de révoquer deux de leurs représentants pour élire Roland et Hérault-Séchelles, Roland dut choisir à son tour. Il donna d'abord sa démission de ministre, puis, la Convention ayant émis le vœu de voir les membres du Conseil rester à leur poste, il revint sur sa décision. C'est au cours de cette séance de la Convention que Danton lança le sarcasme : 
Personne ne rend plus de justice que moi à Roland; mais je vous dirai : $\mathrm{Si}$ vous lui faites une invitation, faites-la donc aussi à Mme Roland; car tout le monde sait que Roland n'était pas seul dans son département. Moi, j'étais seul dans le mien. (Introduction, p. 27)

Dans ses Mémoires, Mme Roland revient sur l'épisode, en le prenant un peu en amont, avant les massacres de septembre 1792 :

Danton et Fabre cessèrent de venir me voir dans les derniers jours d'août [...], ils avaient assez jugé ce qu'étaient Roland et ses entours. [...] Ils conclurent que Roland était un honnête homme, avec lequel il n'y avait rien à faire en entreprises de leur genre; que sa femme n'offrait aucune prise par laquelle on pût influer sur lui; que tout aussi ferme dans ses principes elle avait peut-être de cette sorte de pénétration propre à son sexe dont les gens faux ont à se défier davantage; peut-être aussi augurèrent-ils qu'elle pouvait quelquefois tenir la plume, et qu'en somme un tel couple, fort de raison, de caractère, avec quelques talents, pouvait nuire à leurs desseins et n'était bon qu'à perdre. La suite des événements, éclairés d'ailleurs par une foule de détails qu'il me serait difficile d'exposer aujourd'hui, mais dont il me reste un vif sentiment, donne à ces conjectures toute l'évidence de la démonstration. (p. 117-118)

Elle est consciente des difficultés inhérentes à sa position de femme de ministre, où la dimension privée rejoint la dimension publique : en attaquant l'épouse du ministre, et donc en s'en prenant à sa vie privée, on cherche à le déstabiliser et à le discréditer en tant qu'homme public.

Trois semaines et plus s'étaient écoulées, les journées de Septembre étaient passées; Marat avait eu l'impudence d'afficher la demande des quinze mille livres à d'Orléans, en se plaignant du ministre qui avait eu l'incivisme de ne pas les lui donner, lorsqu'il fit un placard contre moi nommément [L'Ami du peuple, $\mathrm{n}^{\circ} 683$, du 19 septembre 1792]. Je n'y fus pas trompée. «Voilà, dis-je à mon mari du Danton tout pur; il veut vous attaquer, il commence par rôder autour de vous; puis, avec son esprit, il a la bêtise d'imaginer que je serai sensible à ces sottises, que je prendrai la plume pour y répondre; qu'il aura le plaisir de traduire une femme sur la scène, et de jeter ainsi du ridicule sur l'homme public à qui je suis attachée. Ces gens-là peuvent avoir quelque opinion de mes facultés, mais ils ne sauraient juger mon âme; ils n'ont qu'à me calomnier tant qu'il leur plaira, ils ne me feront pas bouger, ni me plaindre, ni m'en soucier. » (p. 134-135)

Cependant, la distinction entre le privé et le public, dont Mme Roland cherche à faire croire qu'elle était extrêmement nette et tranchée dans sa vie et dans son couple, était en réalité beaucoup plus floue, puisqu'elle a joué un rôle politique dans la vie publique du ministre Roland, ne seraitce que par l'écriture. Elle cherche à désamorcer l'accusation de Danton, quand elle rapporte à sa manière ses propos, que toute leur contextualisation tend à discréditer : 
[À l'Assemblée] on opina pour qu'il [Roland] fût invité à rester au ministère. [...] Il [Danton] s'éleva avec beaucoup de chaleur contre cette invitation; son impétuosité trahit sa haine, lui fit dire beaucoup de choses ridicules et, entre autres, qu'il faudrait donc aussi m'adresser l'invitation parce que je n'étais pas inutile au ministère de Roland. (p. 136-137)

Sainte-Beuve confirme, dans ses Portraits de femmes, le rôle politique majeur joué par Mme Roland : «Revenue à Paris à la fin de l'année 91, Mme Roland entra, on peut le dire, au ministère avec son mari en mars 921. » Il ajoute dans une note : « Mme de La Fayette, dans son généreux désir d'aller au secours du général captif, avait fini par avoir pour appui sincère Roland, c'est-à-dire Mme Roland ${ }^{2}$. »

Il s'agit dans ce texte d'un exercice compliqué, où une femme exceptionnelle, par son éducation, son intelligence, son goût pour l'écriture, et son mariage avec un homme qui deviendra ministre, cherche à prouver qu'elle n'a joué aucun rôle, et à se présenter comme une épouse banale prise dans la tourmente de l'Histoire. Ces Mémoires oscillent sans cesse entre une affirmation de la différence des sexes et de leurs rôles respectifs, et une revendication secrète d'une égalité entre eux, sinon dans les faits, du moins dans les potentialités intellectuelles. Le lecteur se trouve pris dans un tourniquet très particulier, dans lequel Mme Roland affirme sa spécificité de femme tout en la niant. C'est ainsi que, lorsqu'elle arrive à l'Assemblée pour dénoncer la tentative d'arrestation de Roland, Vergniaud lui dit :

Dans l'état où est l'Assemblée, je ne puis vous flatter, et vous ne devez guère espérer; si vous êtes admise à la barre, vous pourrez comme femme obtenir un peu plus de faveur; mais la Convention ne peut plus rien de bien. (p. 53)

À son retour elle est arrêtée par un brigadier :

\footnotetext{
Une femme à cette heure, c'est inconcevable; c'est bien imprudent ! [...] — Mais, Madame, toute seule? — Comment, Monsieur, seule! Ne voyezvous pas avec moi l'innocence et la vérité : que faut-il de plus? (p. 57)
}

Quand elle revient sur les premières années de son mariage, elle raconte comment, au printemps 1784, alors que le couple habite Amiens, elle apprend que l'inspection de Lyon vient d'être donnée à un très jeune homme, et elle sollicite cette place pour Roland :

1. Édition de Gérald Antoine, Gallimard, Folio, 1998, p. 231.

2. Ibid. Voir p. 248 : « On a voulu, dans ces derniers temps, faire de Mme Roland un type pour les femmes futures, une femme forte, républicaine, inspiratrice de l'époux, égale ou supérieure à lui, remplaçant par une noble et clairvoyante audace la timidité chrétienne, disait-on, et la soumission virginale. » Il cite une lettre de la jeune Manon à Sophie Cannet : "Quelquefois je suis tentée de prendre une culotte et un chapeau, pour avoir la liberté de chercher et de voir le beau de tous les talents » (p. 265). 
Les commissions étaient déjà expédiées; je fis valoir mes raisons avec l'avantage qu'une femme avait encore dans ce temps-là près des gens qui se piquaient de politesse; on me fit valoir les difficultés, que j'appréciai librement ce qu'elles valaient, et j'obtins le changement presque en même temps que l'annonce faite à mon mari de la demande que j'avais imaginé d'en faire. (p. 171)

Cette républicaine qui «pleurai[t] à douze ans de n’être pas née Spartiate ou Romaine » et a « cru voir dans la Révolution française l'application inespérée des principes dont [elle s'] étai[t] nourrie » (p. 75), semble exprimer ici une sorte de nostalgie pour l'Ancien Régime et pour le statut que la «politesse » y réservait aux femmes. C'est une façon détournée de critiquer la grossièreté des calomnies et des injures dont elle est victime. La meilleure défense qu'elle puisse construire, c'est l'attaque. Elle ébauche ainsi un contre-modèle de ce qu'elle a été, et de l'intrication immorale et blâmable du privé et du public, du masculin et du féminin, du politique et du sexuel :

Le bruit se répandit de je ne sais quelle place accordée ou quelle affaire arrangée par Bonnecarrère, au prix de cent mille livres dont partie devait être remise à Mme de Beauvert. C'était la maîtresse de Dumouriez, vivant chez lui où elle faisait les honneurs de sa table, au grand scandale des hommes sensés, des amis des mœurs et de la liberté. Car cette licence de la part d'un homme public chargé d'affaires d'État marque trop bien le mépris des bienséances, et Mme de Beauvert, sœur de Rivarol, très connu sous un mauvais jour, était environnée de suppôts de l'aristocratie, fort peu recommandables à tous égards. (p. 100)

Où l'on voit apparaître toute la différence entre la « maîtresse » et l'épouse légitime, entre la « licence » de la table et les repas réglés servis chez Mme Roland à l'entourage respectable de son député puis ministre de mari. Elle consacre de longs passages justificatifs à l'économie domestique de ces réceptions, et à son rôle de femme de ministre qui ne veut pas outrepasser les limites où la maintient son statut de femme. Avant même l'entrée de Roland au ministère, elle raconte leur arrivée à Paris le 20 février 1791 et les amitiés qui se nouent :

Brissot vint nous visiter [...]. Il nous fit connaître ceux des députés que d'anciennes relations ou la seule conformité des principes et le zèle de la chose publique réunissaient fréquemment pour conférer sur elle. Il fut même arrangé que l'on viendrait chez moi quatre fois la semaine dans la soirée, parce que j'étais sédentaire, bien logée, et que mon appartement se trouvait placé de manière à n'être fort éloigné d'aucun de ceux qui composaient ces petits comités.

Cette disposition me convenait parfaitement; elle me tenait au courant des choses auxquelles je prenais un vif intérêt; elle favorisait mon goût pour suivre les raisonnements politiques et étudier les hommes. Je savais quel rôle convenait à mon sexe, et je ne le quittai jamais. Les conférences se tenaient en ma présence sans que j'y prisse aucune part; 
placée hors du cercle et près d'une table, je travaillais des mains, ou faisaient des lettres, tandis que l'on délibérait; mais eussé-je expédié dix missives, ce qui m'arrivait quelquefois, je ne perdais pas un mot de ce qui se débitait, et il m'arrivait de me mordre les lèvres pour ne pas dire le mien. (p. 91-92)

Cette page est particulièrement intéressante car elle met en place, de façon presque caricaturale, le « rôle » de Mme Roland et les limites dont elle est consciente. Mais en même temps, il s'agit d'affirmer que, tenue « hors du cercle » par des règles à la fois extérieures, sociales, et totalement intériorisées, elle y aurait tout à fait eu sa place, que de fait elle a eue, ce qu'il lui faut démentir. Dans un même mouvement, elle affirme sa spécificité de femme et les limites qu'elle induit, et la nie. Tourniquet infernal qui prend le lecteur au piège, après avoir entraîné la mort de son habile instigatrice. Elle se met ainsi en scène dans les seules activités tolérées pour les femmes à l'époque : travail manuel d'abord, ou, s'il s'agit d'un travail intellectuel, il sera limité à la correspondance, puisqu'on a longtemps cantonné les femmes dans le genre épistolaire, considéré comme inférieur et plus intime, et donc plus propre à leurs talents particuliers, à leurs prédispositions à l'expression de l'intériorité, et à leur infériorité intellectuelle longtemps tenue pour une vérité allant de soi.

Elle revient longuement sur cette question un peu plus loin, dans les « Portraits et anecdotes », et dans des termes presque similaires :

Là, on examinait l'état des choses, celui de l'Assemblée, ce qu'il conviendrait de faire, comment on pourrait le proposer, les intérêts du peuple, la marche de la cour, la tactique des individus. Ces conférences m'intéressaient beaucoup, et je ne les aurais pas manquées, quoique je ne m'écartasse jamais du rôle qui convenait à mon sexe.

Assise près d'une fenêtre, devant une petite table sur laquelle étaient des livres, des objets d'étude, de petits ouvrages de mains, je travaillais ou je faisais des lettres tandis que l'on discutait. Je préférais écrire, parce que cela me faisait paraître plus étrangère à la chose et m'y laissait presque aussi bien; je puis en faire plus d'une à la fois, et l'habitude du genre épistolaire me permet d'entretenir ma correspondance en écoutant tout autre chose que ce que j'écris; il me semble que je suis trois; je partage mon attention en deux comme une chose toute matérielle, et je considère et dirige l'emploi de ces deux parts comme si j'étais une autre. [...] Excepté les compliments d'usage à l'arrivée et au départ de ces Messieurs, je ne me permis jamais de prononcer un mot, quoique j'eusse souvent besoin de me pincer les lèvres pour m'en empêcher. Si quelqu'un m'adressait la parole, c'était après le cercle rompu et toute délibération terminée. Du reste, une carafe d'eau, un sucrier, était l'unique rafraîchissement qu'on trouvât chez moi, et j'annonçais que c'était le seul qu'il me parût convenable d'offrir à des hommes qui venaient discuter en sortant de table. J'ai réfléchi souvent, mais particulièrement à cette époque, combien le sage Pythagore avait profondément combiné l'effet du silence et avec quelle raison il en faisait faire un noviciat à ses disciples. (p. 197-198) 
Cette stricte économie et cet éloge du silence par une femme, alors qu'on associe fréquemment le sexe féminin à la frivolité et au bavardage, permettent de prendre à rebours certaines idées reçues et d'affirmer sa singularité tout en maintenant le cadre strict des bienséances, dans le sentiment de ce qu'on doit, à soi et aux autres. Elle définit la politique au sens noble comme « l'art de gouverner les hommes et d'organiser leur bonheur en société », et précise immédiatement :

Personne n'en parle moins que moi, parce que la discussion sied peu aux femmes et que celles qui traitent d'affaires d'État me paraissent toujours ressembler à de vieilles gazettes, mais rien à mes yeux n'est plus digne de méditation. Je ne me permets d'en entretenir quelquefois que mes amis; je laisse causer les autres, je me plais assez avec certains vieillards qui s'imaginent instruire tous ceux à qui ils parlent et qui pourraient me voir souvent sans croire que je susse autre chose que coudre une chemise et faire une addition. (p. 198-199)

Là encore, il s'agit d'affirmer une compétence exceptionnelle pour une femme, sans sortir des limites qui lui sont imparties en tant que femme. Rien de tel que l'humour pour réussir un tel tour de force rhétorique... D'autant plus qu'elle parle comme un homme, et avec les mêmes préjugés qu'eux contre les femmes, quand elle évoque l'incompétence d'un collègue de son mari :

Je n'ai jamais bien compris ce qui avait pu faire estimer Duranthon capable d'entrer au ministère, si ce n'est l'idée du peu de facultés nécessaires pour remplir celui de la Justice. Lourd, paresseux, vain et parleur, timide et borné, ce n'était véritablement qu'une vieille femme. (p. 246)

Ce qui valait pour la femme du député sert de principes à la femme du ministre de l'Intérieur lorsque, sous l'Assemblée législative, il entra dans le cabinet girondin formé en mars 1792. Elle justifie ainsi ses réceptions : « Il $\mathrm{y}$ avait Conseil quatre fois la semaine; les ministres convinrent de manger ensemble chez l'un d'eux le jour de ses séances; je les recevais tous les vendredis » (p. 97). La construction de la phrase est tout à fait révélatrice : tout découle du pouvoir, de la raison d'État, des nécessités de service. La première personne n'intervient qu'après la référence au Conseil et aux ministres. Tout un ordre protocolaire est donc respecté dans la syntaxe elle-même, et la succession des propositions suit la diffusion du pouvoir et vaut comme principe de légitimation du « je » et de ses actions. Elle écarte les femmes de ses relations, comme si elle sentait confusément qu'elle ne réussira à s'imposer, ou à faire excuser sa présence comme femme, que comme hapax, et non au sein d'un cercle féminin. Mme Roland fournit tous les détails de sa vie domestique, dans une perspective d'autojustification, où elle cherche à prouver qu'elle n'est jamais sortie du rôle réservé à son sexe, à cette époque, ce qui est pour le moins sujet à caution, comme le prouvent les entortillements de sa démonstration : 
Lorsque mon mari fut au ministère, je m'imposai la loi de ne faire ni recevoir de visites et de n'inviter à manger aucune femme; je n'avais pas de grands sacrifices à faire à cet égard, car n'étant point de résidence habituelle à Paris, mon cercle n'y était pas fort étendu; d'ailleurs je ne m'étais livrée nulle part à la grande société, parce que j'aime l'étude autant que je hais le jeu et que je m'ennuie des sots. Habituée à passer mes jours dans l'intérieur de mon domestique, je partageais les travaux de Roland et je cultivais mes goûts particuliers. C'était donc à la fois conserver ma manière d'être, et prévenir les inconvénients dont une foule intéressée environnent les personnes qui tiennent aux grandes places, que d'établir cette sévérité dans mon hôtel. Je n'y ai jamais eu proprement de cercle de société; je recevais à dîner deux fois la semaine des ministres, des députés, celles des personnes avec lesquelles mon mari avait besoin de s'entretenir ou de conserver des relations. On causait d'affaires devant moi, parce que je n'avais ni la manie de m'en mêler, ni d'entourage qui inspirât la défiance. De toutes les pièces d'un vaste appartement, j'avais choisi pour l'habiter journellement le plus petit salon formant cabinet, où $\mathrm{j}$ 'avais mes livres et un bureau. Il arrivait souvent que des amis ou des collègues ayant besoin de parler confidentiellement au ministre, au lieu d'aller chez lui, où ses commis et le public l'environnaient, se rendaient chez moi et le priaient de l'y faire appeler. Je me suis ainsi trouvée dans le courant des choses sans intrigue ni vaine curiosité : Roland y avait l'agrément de m'en entretenir ensuite dans le particulier avec cette confiance qui a toujours régné entre nous, et qui y a mis en communauté nos connaissances et nos opinions; il arrivait aussi que les amis qui n'avaient qu'un avis à communiquer, un mot à dire, toujours certains de me trouver, s'adressaient à moi pour me charger de lui rendre au premier instant. (p. 106-107)

\section{Évoquant le renvoi de Roland en juin 1792, elle écrit :}

Nous voilà donc rentrés dans la vie privée. On me demandera peut-être si je n'ai jamais eu plus de détails sur la manière dont Roland avait été appelé au ministère? Je puis affirmer que non, et que même je n'ai pas eu la pensée de m'en informer. (p. 105)

\section{De même à propos de « la révolution du 10 août » :}

[J]e n'en sais pas plus que le public à cet égard; car instruite de la grande marche des affaires tant que Roland a été homme public, et la suivant avec intérêt même lorsqu'il n'était plus en place, je n'ai jamais été confidente de ce qu'on peut appeler les petites manœuvres, de même qu'il n'a jamais été agent de cette espèce. (p. 110)

\section{La démarche d'autojustification se fait plus nette encore dans l'évocation} du retour de Roland au ministère :

Au second ministère de Roland, comme au premier, je m'étais imposé de ne recevoir chez moi aucune femme, et j'ai suivi scrupuleusement cette règle. [...]

Le goût et la propreté régnaient sur ma table, sans profusion, et le luxe des ornements n'y parut jamais; on y était à l'aise, sans y consacrer 
beaucoup de temps, parce que je n'y faisais faire qu'un service [...]. Quinze couverts étaient le nombre ordinaire des convives, qui ont été rarement dix-huit et une seule fois vingt. Tels furent les repas que les orateurs populaires traduisirent à la tribune des Jacobins en festins somptueux où, nouvelle Circé, je corrompais tous ceux qui avaient le malheur de s'y asseoir. [...]

Les autres jours, fermés en famille, nous étions souvent, mon mari et moi, tête-à-tête, car, la marche des occupations portant fort loin l'heure du dîner, ma fille mangeait dans sa chambre avec sa gouvernante. (p. 254-255)

L'impression produite, dans ce texte défensif, est celle d'une vie consacrée au travail et à l'utilité publique. Elle entre dans des détails domestiques, non par complaisance féminine à entretenir le lecteur de son intérieur, mais au contraire, par la stricte nécessité de rendre compte de ses fonctions de femme de ministre qui, sans être officielles, lui imposaient certains devoirs de maîtresse de maison, dans la plus stricte économie et la plus grande bienséance, dût sa vie de famille en souffrir.

Elle cherche à faire coïncider son innocence, son ignorance des événements politiques, et son statut de femme, comme le montre ce passage de l'« Interrogatoire de Mme Roland écrit par elle-même » : "puisque n'étant qu'une femme, je n'avais point à me mêler [des affaires] » (p. 141). Elle date le début de la rédaction des « Portraits et anecdotes » à la prison de Sainte-Pélagie le 8 août 1793 et note :

Il y a plus de deux mois que je suis incarcérée parce que j'appartiens à un homme de bien qui s'est avisé d'être vertueux dans une révolution, et de rendre des comptes rigoureux étant ministre. (p. 146)

C'est donc uniquement son statut d'épouse de Roland, marqué fortement par le verbe « appartenir », qui serait en cause, et non sa singularité individuelle, qu'elle ne cesse de rappeler par ailleurs. Pour normaliser sa figure de femme, elle se présente également comme une mère, même si ses réflexions sur la maternité sont rares et assez ambiguës, puisqu'elles reviennent finalement à une revendication de sa sensibilité particulière et du sort qu'elle occasionne :

J'ai une jeune fille aimable, mais que la nature a faite froide et indolente; je l'ai nourrie, je l'ai élevée avec l'enthousiasme et les sollicitudes de la maternité; $[\ldots]$ elle ne connaîtra ni mes vives affections, ni mes peines, ni mes plaisirs; et cependant, si j'avais à renaître avec le choix des dispositions, je ne voudrais pas changer d'étoffe, je demanderais aux dieux de me rendre celle dont ils m'ont formée. (p. 59)

Le sentiment maternel ne sert ici que de prétexte pour proclamer sa singularité de femme " vive », de cœur comme d'esprit. Le cri de la nature semble sonner plus juste quand elle relate sa « Seconde arrestation» le 24 juin 1793, alors qu'elle avait été libérée le matin de la prison de l'Abbaye. 
Elle évoque d'abord l'éducation à donner à sa fille Eudora, avant d'être gagnée par la douleur de la séparation :

Aussi l'étude des beaux-arts, considérée comme partie de l'éducation chez les femmes, doit, ce me semble, avoir moins pour objet de leur faire acquérir un talent distingué que de leur inspirer le goût du travail, leur faire contracter l'habitude de l'application, et de multiplier leurs moyens d'occupation; car c'est ainsi qu'on échappe à l'ennui, la plus cruelle maladie de l'homme en société; c'est ainsi qu'on se préserve des écueils du vice, et même des séductions bien plus à craindre que lui.

Je ne ferai point de ma fille une virtuose [...].

Mais, bon Dieu! je suis prisonnière, et elle vit loin de moi! je n'ose même pas la faire venir pour recevoir mes embrassements; la haine poursuit jusqu'aux enfants de ceux que la tyrannie persécute, et le mien paraît à peine dans les rues avec ses onze ans, sa figure virginale et ses beaux cheveux blonds, que ces êtres apostés pour le mensonge ou séduits par lui le font remarquer comme le rejeton d'un conspirateur. Les cruels! comme ils savent bien déchirer un cœur de mère!

L'aurais-je fait venir avec moi? Je n'ai pas encore dit comment on est à Sainte-Pélagie. (p. 273-274)

Ce qui frappe, dans ce récit qui pourrait être très pathétique, c'est la volonté de se tenir au-dessus des émotions, de les maîtriser, de ne pas donner raison à ceux qui voudraient associer ce qui ne s'appelle pas encore l'hystérie et la féminité. C'est ainsi qu'elle raconte son arrivée à la prison de l'Abbaye :

La femme du concierge qui me parlait ainsi ajoute quelques expressions obligeantes sur les regrets qu'elle forme toutes les fois qu'elle voit arriver des personnes de mon sexe. « Car, ajoute-t-elle, toutes n'ont pas l'air serein comme madame. » Je la remercie en souriant; elle m'enferme. [...]

«Puis-je écrire, puis-je voir quelqu'un? quelle est la dépense à faire ici? » Ce furent mes premières questions. (p. 65)

On voit que la question de l'écriture est centrale et qu'elle arrive en premier. Ce n'est pas un hasard, même si on pourrait la rapporter à un trait typiquement féminin, Mme Roland l'associant parfois aux activités manuelles propres aux femmes. C'est bien là toute l'ambiguïté, car chez elle, l'écriture, et même l'écriture des lettres, acquiert une dimension d'habitude réservée aux hommes : réflexion, persuasion, argumentation, débat politique. Elle donne d'ailleurs juste après un extrait de sa lettre du $1^{\text {er }}$ juin 1793 à la Convention nationale (p. 68). Et, comme s'il fallait confirmer le caractère pour ainsi dire viril du maniement de l'écriture chez elle, elle précise qu'elle a demandé à son arrivée dans cette prison « les Vies des hommes illustres de Plutarque, qu'à l'âge de huit ans [elle] portai[t] déjà à l'église au lieu d'une Semaine Sainte » (p. 72). Or c'est précisément ses talents d'écriture politique qui l'ont rendue suspecte, en lui faisant prendre une grande part aux actions et aux décisions de Roland. 
Quand il est nommé au «comité de correspondance de la Société [des Jacobins] » (p. 219), elle y joue de fait un rôle très actif, à sa place :

[J]e m'occupais de cette correspondance avec plaisir, et le comité trouvait Roland travailleur; il n'était pas non plus sans rien faire, mais l'ouvrage de deux personnes très expéditives devait être considérable aux yeux de ceux à qui l'ouvrage d'une d'elles aurait déjà paru l'être. (p. 221)

Quand Louis XVI se refuse à sanctionner les décrets du 27 mai 1792, sur la déportation des prêtres réfractaires, et celui du 8 juin, sur la formation d'un camp de vingt mille gardes nationaux sous Paris, les trois ministres girondins se rebellent, et Roland prend l'initiative d'envoyer au roi une mise en demeure le 10 juin. Cette lettre a été entièrement rédigée par Mme Roland et montre combien son influence fut alors décisive. Le 13 juin, Roland, Clavière et Servan reçoivent leur congé. À la faveur de la journée du 10 août 1792, Roland revient au ministère. Mme Roland reconnait elle-même : «Je fis la fameuse lettre » (p. 232). C'est alors qu'elle s'arrête longuement sur les accusations dont elle a été victime et qui expliquent son arrestation, dans une page aux explications assez entortillées, où la question de l'articulation entre le pouvoir et son statut de femme est centrale :

Je m'arrête ici un moment pour éclairer les doutes et fixer l'opinion de beaucoup de personnes dont la plupart ne m'attribuent quelque mérite que pour l'ôter à mon mari, et dont plusieurs autres me supposent avoir eu dans les affaires un genre d'influence qui n'est pas le mien. L'habitude et le goût de la vie studieuse m'ont fait partager les travaux de mon mari tant qu'il a été simple particulier; j'écrivais avec lui, comme j'y mangeais, parce que l'un m'était presque aussi naturel que l'autre, et que, n'existant que pour son bonheur, je me consacrais à ce qui lui faisait le plus de plaisir. Il décrivait des Arts, j'en décrivais aussi, quoiqu'ils m'ennuyassent; il aimait l'érudition, nous faisions des recherches; il se délassait à envoyer quelque morceau littéraire à une académie, nous le travaillions de concert ou séparément, pour comparer ensuite et préférer le meilleur ou refondre les deux; il aurait fait des homélies, que j'en aurais composé. Il devint ministre: je ne me mêlai point de l'administration; mais s'agissait-il d'une circulaire, d'une instruction, d'un écrit public et important, nous en conférions suivant la confiance dont nous avions l'usage, et, pénétrée de ses idées, nourrie des miennes, je prenais la plume que j'avais plus que lui le temps de conduire. Ayant tous deux les mêmes principes et un même esprit, nous finissions par nous accorder sur le mode, et mon mari n'avait rien à perdre en passant par mes mains. Je ne pouvais rien exprimer, en fait de justice et de raison, qu'il ne fût capable de réaliser ou de soutenir par son caractère et sa conduite, et je peignais mieux qu'il n'aurait dit ce qu'il avait exécuté ou pouvait promettre de faire. Roland sans moi n'eût pas été moins bon administrateur; son activité, son savoir sont bien à lui, comme sa probité; avec moi il a produit plus de sensation, parce que je mettais dans ses écrits ce mélange de force et de douceur, d'autorité de la raison et de charmes du sentiment qui n'appartiennent peut-être qu'à une femme 
sensible douée d'une tête saine. Je faisais avec délices ces morceaux que je jugeais devoir être utiles, et j'y trouvais plus de plaisir que si j'en eusse été connue pour l'auteur. (p. 233)

Cette tentative de justification n'a guère convaincu les historiens, et Mme Roland semble avoir joué un rôle bien plus grand que celui qu'elle veut bien reconnaitre. On trouve ainsi les informations suivantes dans le Robert des noms propres (édition de 1994) :

Installée à Paris avec son mari (1791), elle fut, plus que lui, la conseillère et l'âme du mouvement girondin, dont les membres, tels Barbaroux, Buzot, Brissot, Condorcet, Pétion de Villeneuve, se réunissaient dans son salon. Son influence et son rôle furent considérables lors du ministère girondin (mars-juin 1792).

Évoquant les lettres qu'elle a reçues lorsqu'elle était «la femme d'un ministre » et qui provenaient «de parents et d'admirateurs, dont [elle] n'avai[t] jamais entendu parler », et qu'elle a éconduits en arguant de son peu d'influence et de pouvoir auprès de son mari, elle constate, non sans une certaine mauvaise foi, semble-t-il, ce paradoxe de sa situation, et sans doute de toute situation de pouvoir :

Ainsi, dans le particulier, j'étais molestée pour ma constance à demeurer concentrée dans mes devoirs, et, dans le public, j'étais calomniée par l'envie, comme si j'eusse dirigé toutes les affaires. Et l'on croit bien doux et bien désirable d'occuper des places éminentes! (p. 187)

Depuis l'anecdote sur le chien recueilli par son cocher, à propos duquel elle note : « je trouvai doux et aimable d'avoir pour cocher à cette heure un bon homme, père et sensible » (p. 56), où l'on reconnaît sans mal l'influence de Diderot et de Rousseau, jusqu'aux détails précis sur son régime alimentaire à la prison de Sainte-Pélagie (p. 270-271), tout est écrit dans la perspective d'un plaidoyer, où elle cherche à apparaître comme une femme de son siècle, sensible et intelligente, et victime de la calomnie, en particulier des montagnards. Mais elle se trahit parfois, montrant qu'elle a eu dans les événements une part beaucoup plus active que le retrait dans lequel elle aime à se mettre en scène. Avant de faire le récit très allusif de l'affaire du Champ-de-Mars le 17 juillet 1791, elle note que, deux jours avant, elle était « au Champ de la Fédération, où la curiosité [1]'avait conduite » (p. 204). Elle rapporte ainsi à un défaut traditionnellement associé au sexe féminin, une soif d'informations sur les événements politiques et leurs grandes manifestations, ce qui est soit une manière de se trahir, soit une façon habile de dissimuler cet investissement. L'affaire du Champ-de-Mars fait suite à la fuite manquée du roi à Varennes en juin 1791 qui eut pour conséquence le développement d'un mouvement démocratique. Sous l'impulsion de certains représentants du Club des jacobins et surtout du Club des cordeliers, une pétition fut présentée à l'Assemblée, exigeant la déchéance du roi; une seconde, demandant son jugement, fut 
déposée par des manifestants sur l'autel du Champ-de-Mars, où avait été célébrée la Fête de la Fédération un an plus tôt. À la suite d'un incident, Bailly proclama la loi martiale, et, au commandement de La Fayette, les gardes nationaux tirèrent sur les manifestants. Cet événement, qui ruina la popularité de La Fayette, accentua les divisions politiques au sein de l'Assemblée constituante et du Club des jacobins dont les modérés, partisans du maintien de la monarchie constitutionnelle, se séparèrent pour former le Club des feuillants.

À la fin des Mémoires particuliers, Mme Roland ajoute une série de «notes ». Nous faisons une entorse à notre décision de limiter notre corpus aux Mémoires strictement historiques, pour considérer la première de ces notes qui apporte également un éclairage sur notre problématique :

\footnotetext{
S'il m'avait été donné de vivre, je n'aurais plus eu, je crois, qu'une tentation: c'eût été de faire les Annales du siècle, et d'être la Macaulay de mon pays; j'allais dire le Tacite de la France, mais cela ne serait point modeste, et les polissons qui ne se piquent pas de l'être, dans l'autre sens, diraient qu'il me manque pour cela quelque chose. J'ai pris dans ma prison une véritable passion pour Tacite; je ne puis dormir sans avoir lu quelques morceaux de lui; il me semble que nous voyons de même et, avec le temps, sur un sujet également riche, il n'aurait pas été impossible que je m'exprimasse à son imitation. (p. 521)
}

Catharine Macaulay est une historienne, féministe et pamphlétaire anglaise qui a vécu de 1731 à 1791. Son Histoire d'Angleterre, depuis l'avènement de Jacques $I^{e r}$ jusqu'à la révolution a été traduite en français en 1791-1792. Mme Roland l'a donc lue soit dans cette traduction soit directement en anglais. Cette référence, et plus encore celle à Tacite, montrent que, si la légitimité n'est pas atteinte sur le plan de l'action politique dans la Révolution, elle est recherchée, même si c'est sur le mode de l'humour, dans le domaine du récit historique et de l'analyse critique de cette même Révolution. Là encore, la question du genre est déterminante. Si elle n'est pas encore admise aux premiers rôles de l'Histoire, une femme pourrait-elle au moins prétendre à l'écriture de cette Histoire? Mme Roland en propose forcément une vision parcellaire et orientée, dont les historiens ultérieurs ont montré les limites. Pour autant, ses Mémoires valent bien comme un témoignage sur la période, sans échapper aux servitudes et aux défauts d'un texte qui relève en même temps du plaidoyer, comme les passages cités le prouvent, mais aussi du réquisitoire à d'autres moments.

Dans les « Mémoires particuliers » que nous n'avions pas retenus dans notre corpus, Mme Roland décrit la Révolution comme « cette pierre de touche des hommes » (p. 513). Le mot est sans doute ici synonyme du 
genre humain en général, car cette période tourmentée de l'Histoire a également révélé des figures de femmes qui ont cru y voir une possibilité d'agir. Cela est particulièrement vrai de Mme Roland qui, loin de la politique des boudoirs et des alcôves de l'Ancien Régime, a voulu imprimer la marque de son intelligence, de son éducation et de son action dans l'Histoire. Elle l'a payé de sa vie, et la vision qu'elle donne de sa place de femme dans un monde d'hommes, au fil de ses Mémoires, est ambiguë : elle sent que la question est centrale, et met en place tout un discours de légitimation et de justification, dont le lecteur ne peut pas longtemps être dupe, mais qui le convainc au moins du fait que la problématique du genre est déterminante dans l'écriture de ces Mémoires. En cela, ils sont bien caractéristiques de ce que Jean-Clément Martin a appelé La Révolte brisée dans son livre soustitré Femmes dans la Révolution française et l'Empire ${ }^{3}$. La première édition des Mémoires de Mme Roland est publiée au printemps 1795, et sans doute vendue à 12000 exemplaires, grâce à Louvet et à Bosc. L'édition demeure cependant expurgée des sentiments de Mme Roland pour Buzot et de ses professions de foi déistes. Même post mortem et chez les survivants du parti girondin, Mme Roland fait donc l'objet d'une censure, qui prouve à quel point le statut du féminin en politique ne va pas de soi, et fait l'objet aussi bien de débats que de fantasmes.

Anne Coudreuse Université Paris 13 - CENEL, IUF

3. Paris, Armand Colin, 2008. 